\title{
FIRST STUDIES FOR A LOW TEMPERATURE HIGHER-ORDER-MODE ABSORBER FOR THE CORNELL ERL PROTOTYPE*
}

\author{
M. Liepe ${ }^{\dagger}$, B. Barstow, H. Padamsee, Cornell University, Ithaca, NY 14853, USA
}

\section{Abstract}

Cornell University, in collaboration with Jefferson Laboratory, has proposed the construction of a prototype energyrecovery linac (ERL) to study the energy recovery concept with high current, low emittance beams [1]. The beam with a current of up to $100 \mathrm{~mA}$ will excite significant higher-order-mode (HOM) power in the superconducting (s.c.) RF cavities with frequencies up to $100 \mathrm{GHz}$. Strong damping of the HOMs is essential for beam stability and to reduce the HOM losses to a few hundred Watts per meter. To achieve this demanding goal we plan to place RF absorbing material in the beam tubes between the cavities in the linac. However, this will require operating the HOM absorbers at temperatures below $80 \mathrm{~K}$ to simplify the thermal transition to the cavities at $2 \mathrm{~K}$ with low static losses to $2 \mathrm{~K}$. One possible material candidate is ferrite, as it is used at room temperature in the HOM absorbers in the s.c. CESR cavities. In this paper we present experiments performed to study the RF absorption properties of ferrite at cryogenic temperatures in the frequency range from $1 \mathrm{GHz}$ to $15 \mathrm{GHz}$. First results are shown and the resulting HOM damping is evaluated and discussed.

\section{INTRODUCTION}

In high current storage rings strong broadband HOM damping in superconducting cavities has been achieved by using beam-pipe ferrite loads, located at room-temperature; see [2] for CESR and [3] for KEK-B. Adopting the same damping concept for the ERL with RF absorbers between the cavities in a cavity string will require to operate the absorbers at a temperature of about $80 \mathrm{~K}$. This temperature is high enough to intercepted HOM power with good cryogenic efficiency, and $80 \mathrm{~K}$ is low enough to simplify the thermal transitions to the cavities at $2 \mathrm{~K}$. However, the electro-magnetic properties of possible absorber materials like the ferrite TT2-111R [4] were not well known at cryogenic temperatures. Therefore we started a measurement program at Cornell to answer the question if ferrite remains lossy at cryogenic temperatures.

\section{FERRITE RF PROPERTIES AT $110 \mathrm{~K}$}

The complex $\epsilon$ and $\mu$ properties of a ferrite material called TT2-111R were measured at room temperature and at approximately $110 \mathrm{~K}$ in the frequency range 1 to $15 \mathrm{GHz}$. These properties were measured with a vector network analyzer using a technique suggested by Nicholson and Ross [5] and documented by Hewlett Packard [6].

${ }^{*}$ Work is supported by Cornell University.

†mul2@cornell.edu

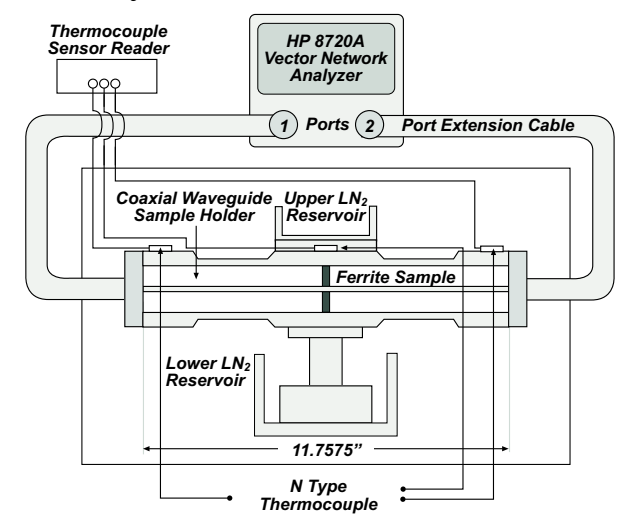

Figure 1: Schematic of low temperature EM properties measurement apparatus.

\section{MEASUREMENT SETUP}

High frequency voltages were applied to a ferrite sample and the amplitude and phase of the reflected and transmitted waves were measured with a vector network analyzer. The normalized amplitude and phase of these waves are called the $S$ parameters. From these $\mathrm{S}$ parameters the complex $\epsilon$ and $\mu$ values of the sample were inferred. A schematic of the experiment is shown in Figure 1. A 12 inch long coaxial waveguide with an outer conductor diameter of $7 \mathrm{~mm}$ and inner conductor diameter of $3 \mathrm{~mm}$ was used to hold the ferrite sample. A coaxial line, unlike a rectangular waveguide can support a wide range of radiation frequencies in a single propagation mode. Our 7 $\mathrm{mm}$ coaxial line can support radiation in a single frequency mode up to $18 \mathrm{GHz}$. To ensure that the microwave reflections from the interface between the cables and waveguide sample holder were minimized, the connector interfaces on the end of the sample holder were carefully machined.

A cooling clamp was attached to the center of the sample holder where the sample is situated. A finger protrudes from the clamp into a bath of liquid nitrogen to cool the sample. ANSYS and analytical calculations indicated that the center of a 12 inch long sample holder may have a temperature close to that of the liquid nitrogen bath $\left(\approx-165^{\circ} \mathrm{C}\right)$ while the ends would be closer to room temperature $\left(\approx-60^{\circ} \mathrm{C}\right)$. This temperature gradient ensured that no damage occurred to the dielectric filled flexible cables that took microwave power from the network analyzer to the sample. It also minimized thermal deformation of the metallic connectors, ensuring minimal change of their physical state from the one they were calibrated at. To prevent ice formation on any of the cooled electronics, the experiment was housed under positive pressure in a nitrogen atmosphere. 


\section{FERRITE SAMPLES}

High quality material samples were needed to achieve reproducible, results with low systematic errors. It is necessary to have very good contact between the ferrite beads and the inner and outer conductors of the coaxial line. Several ferrite beads were carefully fabricated from tiles of TT2-111R. Those that most closely matched the dimensions of the sample holder were selected and used in the experiment.

Beads with an inner diameter slightly too small for the inner conductor were preferred. The inner conductor of the sample holder was cooled with $\mathrm{LN}_{2}$ and the bead was forced along the line to its center. This ensured good contact between the sample and holder at $100 \mathrm{~K}$ and excellent contact at room temperature.

\section{CALIBRATION}

The built in full 2 port calibration algorithm was used to calibrate the network analyzer. The isolation calibration routine was not used because of anecdotal evidence [7] suggesting that the network analyzer set up becomes overly sensitive to movements of the cables when this calibration is performed. It is necessary to correct the phase of the $\mathrm{S}$ parameters recorded by the network analyzer due to additional phase picked up as the microwaves propagate along the sample holder away from the sample. To do this one must know precisely the distance of the sample from the phase reference planes established during the network analyzer calibration. This distance was first measured approximately using a depth gauge. The insertion distance was then refined by comparing the phases of the reflected signals measured at either port of the network analyzer. The two phases should be identical, so the difference between them was minimized by varying the assumed insertion distance of the sample.

\section{MEASUREMENTS AND RESULTS}

A number of room temperature $S$ parameter data sets were then acquired. Gaseous nitrogen was pumped into the box. $\mathrm{LN}_{2}$ was then poured into the reservoirs. The temperature of the middle of the line, where the sample is held, and the ends of the lines were measured. The measurement apparatus was then allowed to cool to its final, quasi equilibrium temperature before $\mathrm{S}$ parameter measurements were taken again.

Due to finite propagation time of signals in the setup and the wide frequency range over which the $S$ parameters were measured it was found that the network analyzer receiver fell out of phase with the transmitter, resulting in an artificially attenuated transmission. To avoid this problem, a wide receiver bandwidth of $3 \mathrm{kHz}$ and slow sweep over a short frequency range were used. Two data sets were taken, with 801 frequency points from 1 to $10 \mathrm{GHz}$, followed by another 801 frequency points from 8 to $18 \mathrm{GHz}$. The two data sets were merged following collection of the data. Good continuity was found between the data sets. It is believed that the presence of the ferrite sample in the line caused excitation of higher order modes above $15 \mathrm{GHz}$, invalidating all measurements of the $\mathrm{S}$ parameters above this frequency.

The $\mathrm{S}$ parameters were converted to complex $\epsilon$ and $\mu$ values following the algorithm outlined by Hartung [8]. Multiple $\mathrm{S}$ parameter data sets spanning the range 1 to $18 \mathrm{GHz}$ were collected at room temperature and $\approx 110 \mathrm{~K}$. The different data sets were then averaged and filtered to smooth out ringing due to microwave reflection from the ends of the sample holder. The final results are shown in Figure 2.

\section{EVALUATION OF HOM DAMPING}

\section{A SIMPLE RF ABSORBER MODEL}

For a first evaluation of the efficiency of the investigated TT2-111R ferrite, we used the simple planar absorber model shown in Figure 3. It is assumed that a TEM incident wave is propagating in positive $z$-direction towards the ferrite material (which extends to infinite in $x$ - and $y$ direction). Based on the measured ferrite properties at 110 $\mathrm{K}$, an absorber efficiency $\eta=P_{\text {absorbed }} / P_{\text {incident }}$ can be calculated, see Figure 4. As can be seen, the absorber is highly efficient at low temperatures in the frequency range in which the material properties have been measured so far.

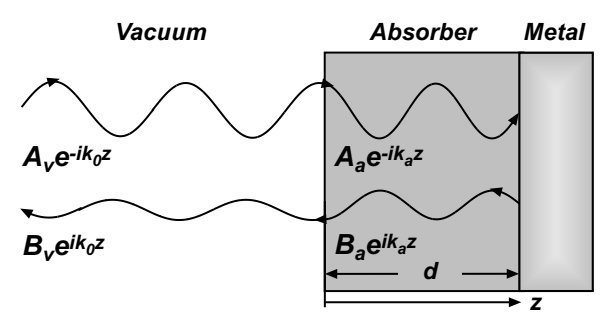

Figure 3: Absorber model.

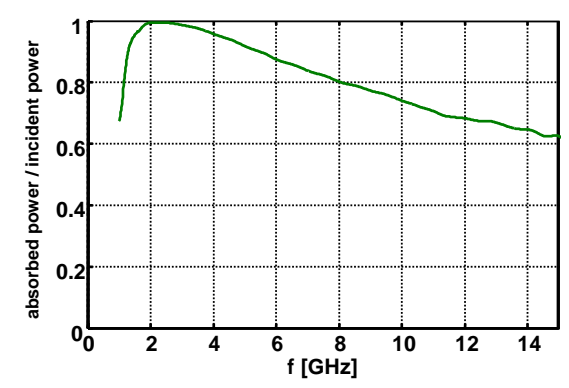

Figure 4: Absorber efficiency for a TT2-111R absorber with $d=2 \mathrm{~mm}$ thickness at $110 \mathrm{~K}$.

\section{ERL INJECTOR CAVITIES}

The finite element code CLANS has been used to calculate the damping of monopole modes by the ferrite absorbers in the injector cavities of the Cornell ERL prototype (refer to [9] for details). CLANS uses a complex eigen solver to calculate modes in an RF structure in the presence 

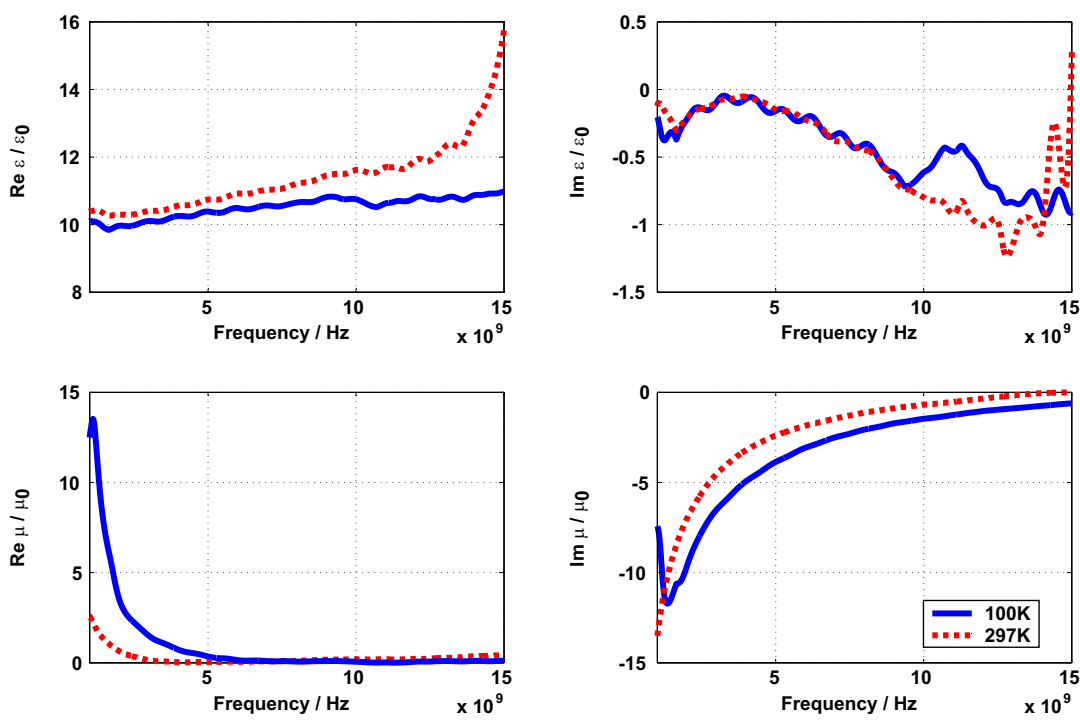

Figure 2: Complex $\epsilon$ and $\mu$ values of TT2-111R.

of an RF absorbing material. The measured complex $\epsilon$ and $\mu$ values of TT2-111R at $110 \mathrm{~K}$ were input into CLANS to describe the absorber properties in the numerical calculations. In the ERL injector the beam will be accelerated by five 2-cell cavities [10]. The presence of a $10 \mathrm{~cm}$ long ferrite ring-absorber between each cavity and at the ends of the cavity string was considered in the simulations. The obtained damping factors, represented via the quality factor $Q=\omega U / P_{\text {diss }}$, are shown in Figure 5. As intended, the fundamental mode passband is not propagating through the tubes, thus the fields at the absorbers are small and these modes are not significant damped by the ferrites. However, all other TM monopole modes are propagating, and their low quality factors show the strong damping by the absorber material.

\section{FUTURE PLANS}

We will extend the measurement of the absorber properties to $40 \mathrm{GHz}$ by using rectangular waveguides as sample holders. Using different size waveguides will ensure that only one propagation mode is supported during a measure-

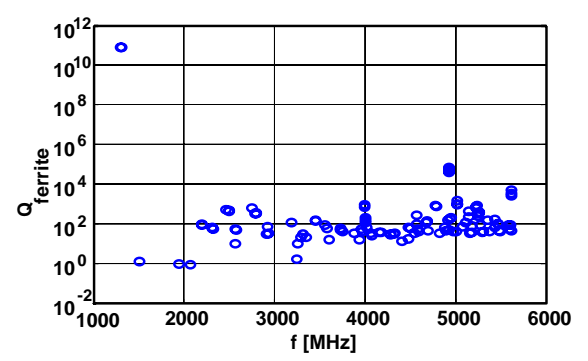

Figure 5: Calculated damping of TM monopole modes by ferrite ring-absorbers in the superconducting injector cavities of the ERL prototype. ment in a certain frequency range. Absorber samples made of different ferrite types have been ordered and their RF losses will be compared to find the optimal broadband RF absorber for the Cornell ERL prototype.

\section{CONCLUSIONS}

The EM properties of the ferrite TT2-111R have been measured at $110 \mathrm{~K}$ and $300 \mathrm{~K}$ in the frequency range 1 to $15 \mathrm{GHz}$. In this frequency range TT2-111R is even more lossy at cryogenic temperatures than at room temperature, and therefore is a promising material for an RF absorber in the Cornell ERL prototype.

\section{ACKNOWLEDGEMENTS}

We would like to thank N. Iqbal, W. Hartung, R. Seeley, N. Sherwood, J. Kaminski, C. Firenze, J. Sears, and R. Miller for invaluable help during these experiments.

\section{REFERENCES}

[1] Study for a proposed Phase I ERL Synchrotron Light Source at Cornell University, ed. by S. Gruner and M. Tigner, CHESS Tech. Memo 01-003, JLAB-ACT-01-04 (July 2001).

[2] D. Moffat et al., in Proceedings of the 1993 Particle Accelerator Conference, Washington, DC, p. 977 (1993).

[3] KEKB B-Factory Design Report, Technical Report 95-7, KEK, Tsukuba, Japan, 1995.

[4] Product of Trans Tech, Adamstown, MD 21710, USA.

[5] A.M. Nicholson and G.F. Ross, IEEE Trans. Instrum. Meas. IM-19, p. 377-382 (1970).

[6] HP Product Note 8510-3: Measuring the dielectric constant of solids with the HP 8510 network analyzer, Hewlett Packard (1985).

[7] S. Ganchev, private communication.

[8] W.H. Hartung, Ph.D. thesis, Cornell University, Ithaca NY (1996).

[9] H. Padamsee et al., this conference, paper TPAB052.

[10] V. Shemelin et al., this conference, paper WPAB012. 